Original Paper http://ajol.info/index.php/ijbcs http://indexmedicus.afro.who.int

\title{
Potentiel de régénération des chantiers de production du charbon de bois au Centre-Bénin
}

\author{
Alain Sèakpo YAOITCHA ${ }^{1,2 *}$, André Boya $\mathrm{ABOH}^{1,3}$, Alex Gbèliho ZOFFOUN ${ }^{1,2}$, \\ Marcel HOUINATO ${ }^{1}$, Guy Apollinaire MENSAH ${ }^{2}$, Brice SINSIN $^{1}$ et \\ Elie Léonard $\mathrm{AKPO}^{4}$
${ }^{1}$ Laboratoire d'Ecologie Appliquée, Faculté des Sciences Agronomiques, Université d'Abomey-Calavi, O1BP 526, Cotonou, Bénin.
${ }^{2}$ Institut National des Recherches Agricoles du Bénin, 01 BP 884, Cotonou, Bénin.
${ }^{3}$ Ecole de Gestion et d'Exploitation des Systèmes d'Elevage de l'Université Nationale d'Agriculture de Porto-Novo, Bénin. \\ ${ }^{4}$ Laboratoire de Biologie Végétale, Faculté des Sciences et Techniques, Université Cheikh Anta Diop, BP 5005, \\ Dakar, Sénégal. \\ *Auteur correspondant; E-mail : ayaoitcha@gmail.com; Tel : (00229) 97778444.
}

\section{RESUME}

Le présent travail a pour objectif d'étudier le potentiel d'émission des rejets des souches des arbres coupés pour la production du charbon de bois. L'inventaire des espèces exploitées a été réalisé dans 24 chantiers de production de charbon répartis dans trois Communes du Département des Collines au Bénin. Au total, 17 essences forestières ont été inventoriées dans les 24 chantiers prospectés. Deux groupes de chantiers ont été formés : le groupe des chantiers sur lesquels une large gamme d'espèces sont exploitées et le groupe assemblant les chantiers quasi-monospécifiques. Les espèces les plus exploitées sont: Burkea africana, Prosopis africana, Anogeissus leiocarpa, Pterocarpus erinaceus, Vitellaria paradoxa et Pseudocedrela kotschyi. Dans ce lot, deux catégories d'espèces ont été identifiées en matière de régénération des souches: celle des espèces dont la survie des souches est assurée à environ $100 \%$ quels que soient l'âge et les dimensions des souches et celle des espèces dont la survie est assujettie aux variations de l'âge et les dimensions des souches. Somme toute, les stratégies de conservation urgentes ont été proposées pour mieux assurer l'utilisation durable de toutes les espèces qui sont devenues la proie de la production du charbon au Bénin. (C) 2016 International Formulae Group. All rights reserved.

Mots clés : Régénération, rejet de souche, survie des souches, production de charbon, Bénin.

\section{The potential of stumps obtained from the sites of charcoal production in Centre of Benin}

\begin{abstract}
This work aims to study the potential of emission of throwing out stumps which were cut for charcoal production. The exploited species were inventoried in 24 sites of charcoal production in three Communes of the Department of the Collines region in Benin. Overall, 17 forest species were inventoried in the 24 sites of charcoal production. Two groups of sites were identified: one constituted by the sites where many species were
\end{abstract}


exploited and the other constituted by the sites where virtually one species where exploited. The most exploited species were: Burkea africana, Prosopis africana, Anogeissus leiocarpa, Pterocarpus erinaceus, Vitellaria paradoxa, and Pseudocedrela kotschyi. Among these species, two categories of tree species were identified regarding the stump regrowth: some which stump survival was ensured at about $100 \%$ whatever age and stumps dimensions and the others which stump regrowth depended on variation of age and dimensions of the stumps. In sum, urgent conservation actions were suggested for improving the sustainable use of all species exploited for charcoal production in Benin.

(C) 2016 International Formulae Group. All rights reserved.

Keywords: Regeneration, stump regrowth, stump survival, charcoal production, Benin.

\section{INTRODUCTION}

Le bois énergie est la principale source d'énergie domestique pour les populations africaines, en particulier au Sud du Sahara. La FAO (2000) a rapporté qu'elles ont exploité plus de 603 millions de mètres cubes de bois, dont $86 \%$ sont consommés sous forme de bois de feu et de charbon de bois.

$\mathrm{Au}$ Bénin, plus de $85 \%$ d'énergie domestique sont fournis par le bois de feu et le charbon de bois extraits en grande partie des formations naturelles (MEPN, 2009). Cette quantité d'énergie utilisée provient en moyenne de 5200000 tonnes de bois de feu et de 19000 tonnes de charbon de bois (Djohossou, 2000; Tchiwanou, 2003). Le prélevement du bois de feu dans ces formations naturelles a certes diminué d'intensité avec le lancement du Projet Plantations de Bois de Feu dans le Sud-Bénin, mais quant à la production du charbon, elle reste dépendante des ligneux sauvages (Yaoitcha, 2007). En effet, la consommation du charbon de bois des ménages a été estimée à $2,6 \%$ en milieu rural et $36,5 \%$ en milieu urbain (PBF II/IFN, 2007). La population urbaine constitue donc le grand consommateur de charbon de bois à cause de la qualité de son combustible, sa manipulation facile, et son acquisition à petite quantité au fur et à mesure de ses besoins. En réponse à la demande exprimée par les populations urbaines du SudBénin, la production du charbon de bois a pris une très grande importance dans certaines régions du Centre-Bénin où la couverture forestière est la moins dégradée comparativement au Sud-Bénin. Les essences forestières exploitées à cet effet sont :
Anogeissus leiocarpa (DC.) Guill. et Perr.; Prosopis africana (Guill et Perr) Taub.; Burkea africana Hook. et Pterocarpus erinaceus Poir. Celles-ci sont devenues de plus en plus rares (Agbani et al., 2004) et elles ne se retrouvent présentement que dans les aires protégées.

Dans les écosystèmes dégradés par l'exploitation tous azimuts dans plusieurs régions du monde: en Amérique (BoivinChabot et al., 2004), en Australie (Forrester et al., 2003), en Nouvelle-Zélande (Sims et al., 2001), et en particulier en Afrique australe (Shackleton, 2000, 2001 ; Kaschula et al., 2005), en Afrique orientale (Luoga et al., 2004) et en Afrique occidentale (Fall et al., 2000; Sawadogo et al., 2002), les investigations ont été faites sur les rejets des souches des arbres coupés pour définir les méthodes d'aménagement et de gestion durable de ces ressources naturelles. Aussi, les travaux scientifiques antérieurs révèlent-t-ils que certaines espèces telles que Pterocarpus erinaceus, Anogeissus leiocarpa, Prosopis africana et Burkea africana, utilisées pour produire du charbon sont capables d'émettre des rejets (Bellefontaine et al., 2000). En prélude aux actions de conservation des espèces les plus importantes recherchées dans la production du charbon au Bénin, il est important que des investigations soient faites sur les chantiers de production pour évaluer l'état des souches des espèces utilisées. Ainsi, le présent travail vise à caractériser les chantiers de production du charbon à travers les espèces recherchées et à déterminer l'effet de l'âge et des dimensions des souches sur la survie des souches de ces espèces. 


\section{MATERIEL ET METHODES Milieu d'étude}

L'étude a été conduite au Bénin dans le Département des collines précisément dans les Communes de Dassa-Zoumè, Bantè et Savè, compris entre $2^{\circ} 20^{\prime}$ et $2^{\circ} 28^{\prime}$ de longitude Est et $7^{\circ} 45^{\prime}$ et $7^{\circ} 52^{\prime}$ de latitude Nord (Figure 1). Les terroirs des trois communes sont soumis à un même type de climat; il s'agit d'un climat de type dit "intermédiaire" entre le subéquatorial maritime (caractérisé par deux saisons sèches et deux saisons humides) et le soudanoguinéen (à une saison pluvieuse et une saison sèche). La pluviosité moyenne est de 1121 $\mathrm{mm} / \mathrm{an}$. La température moyenne est de 27,50 ${ }^{\circ} \mathrm{C}$ avec des valeurs extrêmes de $22{ }^{\circ} \mathrm{C}$ (minima) et $30{ }^{\circ} \mathrm{C}$ (maxima). Les mois les plus chauds sont février et mars, et les plus frais sont juillet et août. On rencontre essentiellement un éventail assez complet de sols allant des sols légers épais et peu ferrugineux aux sols argileux profonds (Dubroeucq, 1977). Deux groupes ethniques sont rencontrés dans le milieu : Les autochtones constitués de deux groupes socioculturels : les Nagots et les Mahi et les groupes issus des migrations récentes à savoir : les Fons, les Yom-Lokpa, les Peulh, les Otamari, les Dendi et les Baatonu. Les Fons constituent les groupes socio - culturels connus dans la production du charbon. Ils sont venus du Zou à la recherche des terres fertiles. La végétation est dominée essentiellement par des savanes arborées à arbustives composées de Vitellaria paradoxa, Parkia bigloboza et Pseudocedrela kotschyi.

\section{Relevé de végétation}

Les relevés de végétation ont été réalisés sur les anciens et nouveaux chantiers de production de charbon. Les différentes souches rencontrées dans des placeaux circulaires de $20 \mathrm{~m}$ de rayon ont été recencées. Ces placeaux ont été installés sur les chantiers qui nous ont été indiqués par les charbonniers. Le nom de l'espèce de chacune des souches a été identifié. La détermination des espèces a été faite soit en se basant sur les feuilles des rejets de souche soit sur l'ecorce de souche. Dans chaque placeau, nous avons relevé les paramètres suivants: hauteur de coupe, circonférence à la hauteur de coupe, état de souche.

\section{Analyse des données}

Afin d'identifier éventuellement des groupes de relevés, l'analyse factorielle des correspondances a été réalisée. Pour cette analyse nous avons utilisé le logiciel SAS. L'importance des espèces exploitées (souches uniquement) a été évaluée à partir des principaux paramètres que sont : l'abondance, la densité, la surface terrière, la dominance, la fréquence, la densité relative, la dominance relative, la fréquence relative et la valeur d'importance (IVI). La valeur d'importance (IVI) est un indice qui permet d'indiquer le rôle structurel d'une espèce dans un groupe. Elle est utilisée pour comparer les groupes entre eux, du point de vue floristique et structurel. Le ratio $\mathrm{A} / \mathrm{F}$ : abondance $(\mathrm{A}) /$ fréquence $(\mathrm{F})$ des souches des différentes espèces a été déterminé pour définir le patron de distribution des espèces de chaque groupe. Un ratio inférieur à 0,025 caractérise une distribution régulière, 0,025 à 0,05 une distribution aléatoire, supérieur à 0,05 une distribution contagieuse (Curtis et Cottam, 1956).

Les données collectées sur les chantiers et sur les souches ont servi à définir les variables suivantes :

- Age des souches: période (en années) écoulée depuis la coupe. Cet âge a été donné par le guide de terrain qui est généralement un des exploitants des chantiers visités.

- Taux de survie des souches: proportion des souches ayant donné de rejets (souches vivantes) par rapport au nombre total de souches observées. 


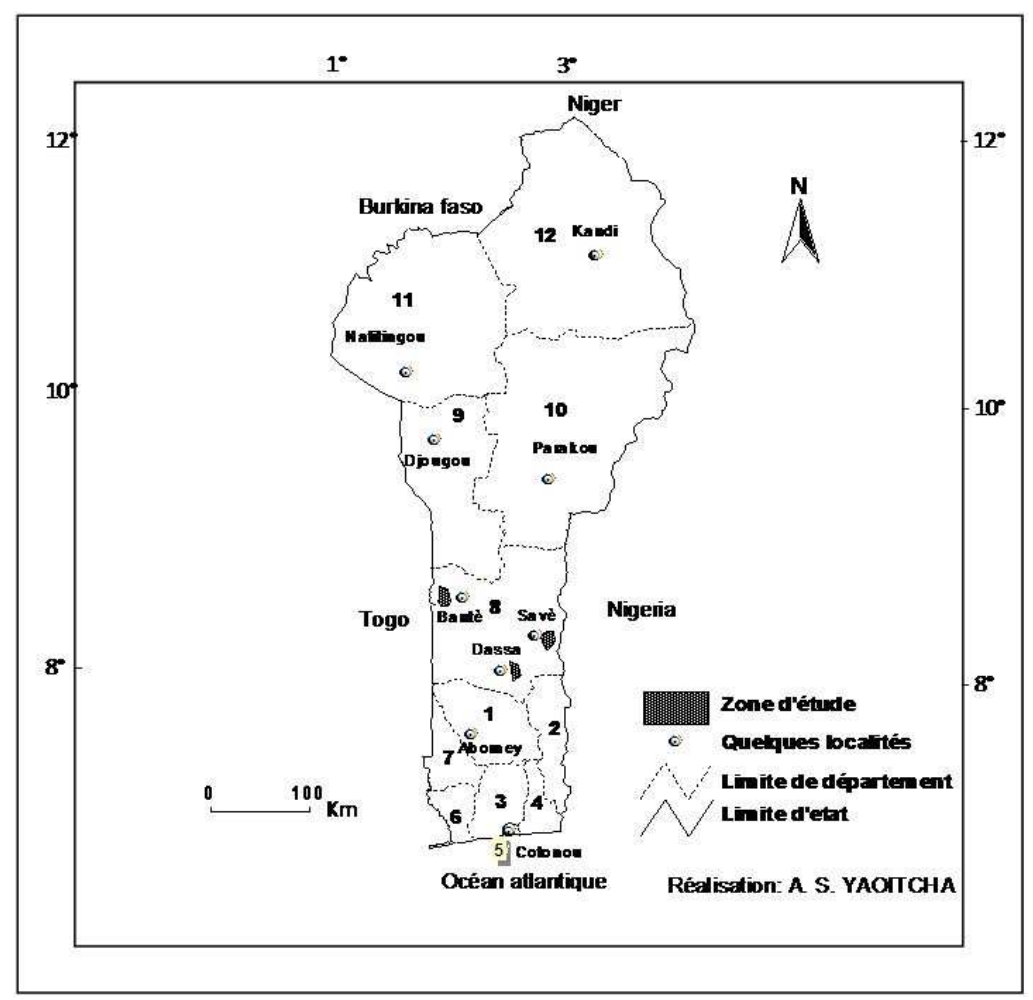

Figure 1 : Localisation de la zone d'étude dans le Bénin

La distribution des taux de survie des souches des espèces en fonction des différentes classes d'âge, de grosseur des souches et de hauteur de coupe a été établie pour mettre en évidence l'influence de ces paramètres sur la survie des souches des espèces. Le test d'égalité des moyennes a été utilisé pour comparer la proportion des souches vivantes et des souches mortes au niveau de chaque variable. Aussi, cette analyse a-t-elle été suivie de l'établissement des modèles de régression linéaire du taux de survie des souches des espèces en fonction de l'âge, la circonférence des souches et la hauteur de coupe afin de prédire la survie des souches des espèces.

\section{RESULTATS}

\section{Composition floristique des chantiers}

Au total, 24 chantiers répartis entre les trois communes ont été prospectés. Sur l'ensemble des 24 chantiers de production de charbon explorés, 17 espèces appartenant à 17 genres, ont été inventoriées. Celles-ci ont été réparties dans 9 familles. Les familles les plus représentées ont été : Leguminosae (6 espèces), Combretacae (3 espèces) et Sapotacae (2 espèces). Ces 3 familles comptent au total plus de la moitié de la flore. Les autres familles ont été représentées par une seule espèce. En fonction de l'effectif et de la fréquence de présence des espèces dans les sites, des groupes d'espèces suivants ont été constitués :

- les espèces préférentiellement exploitées: Anogeissus leiocarpa, Burkea africana, Pterocarpus erinaceus, Vitellaria paradoxa , Pseudocedrela kotschyi et Prosopis africana. Elles ont été présentes au moins dans $25 \%$ des chantiers explorés et comptent environ 30 individus par espèce ; - les espèces indifféremment exploitées : Terminalia spp, Lophira lanceolata, Bridelia ferruginea, Malacantha 
alnifolia, Parinari curatellifolia, Isoberlinia doka et Pericopsis laxiflora. Elles ont été soit présentes sur au moins 2 chantiers ou comptent au moins 2 individus par espèce et

- les espèces accidentellement exploitées: Afzelia africana, Ficus spp, Maytenus senegalensis et Pteleopsis suberosa. Elles ont été représentées par un seul individu dans l'ensemble des chantiers prospectés.

\section{Hétérogénéité floristique des chantiers}

L'analyse factorielle des correspondances a permis de définir l'hétérogénéité floristique des chantiers. L'information contenue au niveau des axes factoriels varie de $0,025 \%$ à $0,716 \%$, soit de $0,62 \%$ à $17,69 \%$ (Tableau 1). Les deux premiers axes factoriels qui définissent le plan principal, ont absorbé $35,1 \%$ de l'inertie totale. Le troisième axe factoriel, qui en a absorbé $15,11 \%$ de l'inertie totale a permis ainsi d'établir l'homogénéité de la distribution dans le plan des axes factoriels F1 et F2. Les espèces et les chantiers qui interviennent de manière significative dans la définition des axes factoriels F1 x F2 ont permis d'identifier les groupes ci-dessous (Figure 2).

En effet, l'axe F1 a opposé les chantiers sur lesquels plusieurs espèces ont été exploitées (B02, D02, D03 et S01) aux chantiers quasi-monospécifiques (B03, B04, D05, S12 et S13). Il peut être interprété comme l'axe du gradient de diversité des espèces exploitées sur les chantiers de charbon. Cet axe F1 a permis de distinguer ainsi deux groupes de chantiers : le groupe à Burkea africana et Parinari curatellifolia (GI) et le groupe à Anogeissus leiocarpa (GII).

L'axe F2 a fait apparaître une subdivision dans le groupe de chantiers à Anogeissus leiocarpa montrant ainsi une certaine hétérogénéité. Nous y avons identifié en effet deux sous-groupes : le sous-groupe à Pseudocedrela kotschyi et Lophira lanceolata (GIIa) et le sous-groupe à Anogeissus leiocarpa et Afzelia africana (GIIb). Anogeissus leiocarpa a fortement contribué dans la définition de cet axe. Ce dernier représente en effet le gradient de distribution de Anogeissus leiocarpa sur les chantiers prospectés.

\section{Caracteristiques structurelles des chantiers}

L'indice d'importance (IVI) des espèces est rapporté dans le Tableau 2. Burkea africana a donné les plus fortes valeurs d'IVI dans le groupe GI suivi de Vitellaria paradoxa qui apparaît comme la seconde espèce dominante du groupe. Maytenus senegalensis et Pteleopsis suberosa ont donné les plus faibles valeurs d'IVI dans le groupe GI. Outre Burkea africana et Vitellaria paradoxa, Prosopis africana et Pterocarpus erinaceus, constituent les seules espèces qui ont donné des valeurs d'IVI supérieures à 25/300. En effet, ces quatre espèces apparaissent les plus importantes du groupe. Dans le groupe GII, Anogeissus leiocarpa a donné la plus forte IVI, suivie de Pterocarpus erinaceus. En somme, Pterocarpus erinaceus, Vitellaria paradoxa et Prosopis africana constituent les principales espèces compagnes de Burkea africana et Anogeissus leiocarpa respectivement des deux groupes GI et GII. Par ailleurs, Pseudocedrela kotschyi est aussi une espèce non moins importante dans l'ensemble des chantiers.

Le patron de distribution des espèces au sein des groupes a été illustré par le ratio Abondance/Fréquence. Les différentes valeurs obtenues indiquent que la majorité des espèces au sein des groupes présentent une distribution régulière. Le groupe GI compte 11 espèces à distribution régulière sur les 12 espèces soit $91,66 \%$ contre $83,33 \%$ pour le groupe GII. Ce dernier compte en plus d'une espèce à distribution aléatoire (Isoberlinia doka), une espèce à distribution contagieuse (Lophira lanceolata). A ce titre, le groupe GI paraît relativement plus homogène que GII.

\section{Capacité de survie ou potentiel de régénération des souches \\ Nous avons dénombré globalement} 247 souches qui ont donné des rejets sur un total de 322 souches d'arbre examinées, soit un potentiel de survie de $77 \%$ (Tableau 3). Le taux de survie des souches de l'ensemble des 
espèces décroît en fonction de leur âge, de leur circonférence et de la hauteur de coupe (Figure 3). Pour les 4 premières années (0 à 4 ans) (Figure 3a), l'équation de la courbe d'ajustement à la distribution du taux de survie a révélé que pour un âge de souche $\mathrm{x}=$ 0 (juste après la coupe des arbres), le taux de survie des souches (y) est environ $100 \%$. A l'opposé, ce taux s'annule $(\mathrm{y}=0 \%)$ pour un âge $x \geq 8$ ans. Ce qui implique qu'après 8 ans il ne serait plus possible d'observer de souches vivantes sur les chantiers de production de charbon. La valeur du coefficient de détermination $\left(\mathrm{R}^{2}\right)$ jointe à l'équation de la courbe d'ajustement est assez élevée $(95,16 \%)$.

Le graphe de la figure $3 b$ montre une baisse du taux de survie des souches des espèces en fonction de la circonférence des souches. Autrement dit, plus l'arbre est gros (vieux) moins la probabilité de survie de sa souche est élevée. L'équation de la courbe d'ajustement indique que le taux de survie (y) s'annule pour un arbre qui mesure environ $475 \mathrm{~cm}$ de circonférence $(\mathrm{x})$. En réalité, toutes les espèces étudiées ne sont pas du genre à atteindre une si grande taille. Les plus gros arbres (200 à $300 \mathrm{~cm}$ de circonférence) ont été relevés au sein des individus de Anogeissus leiocarpa. En revanche, le maximum du taux de survie (y) qu'on puisse atteindre est d'environ 95\%. Mais ce taux n'est obtenu qu'avec un arbre de grosseur nulle $(\mathrm{x}=0)$. En fait, Le plus petit arbre relevé a mesuré $12 \mathrm{~cm}$ de circonférence. En outre, la grosseur minimale d'un arbre est environ $10 \mathrm{~cm}$. Sur cette base, le taux de survie maximum serait égal à 93,38\%. L'équation de cette courbe d'ajustement a été caractérisée par un coefficient de détermination qui est égal à $88,01 \%$.

Les différentes valeurs du taux de survie des souches obtenues dans les classes de hauteur de coupe montrent que plus les arbres sont coupés à une hauteur élevée, moins leur chance de survie est élevée (Figure $3 c)$. La courbe de tendance linéaire associée à cette variation est caractérisée par un coefficient de détermination $\left(\mathrm{R}^{2}\right)$ égal à
97,26\%. Le maximum du taux de survie (y) que l'on puisse obtenir est égal à $90 \%$ pour une coupe rase $(x=0 \mathrm{~cm})$. La hauteur de coupe (x) qui annule le taux de survie est égal à 4,57 $\mathrm{m}$. Ce dernier cas reste théorique. En pratique, la plus grande hauteur de coupe enregistrée est égale à $1,50 \mathrm{~m}$. Le taux de survie correspondant est égal à 60,55\% .

Cette analyse de distribution du taux de survie en fonction de l'âge et les dimensions des souches des espèces dont la quasi-totalité des souches émettent des rejets apparaît moins pertinente. En effet, parmi les principales espèces exploitées, il y en a dont les souches ont presque toutes émis des rejets : Prosopis africana (100\%), Burkea africana (99\%) et Pseudocedrela kotschyi (97\%). Quant aux autres principales espèces (Anogeissus leiocarpa -59\%-, Pterocarpus erinaceus $-67 \%$ - et Vitellaria paradoxa -67\%-) dont les taux de survie des souches remarquablement inférieurs à $100 \%$, les tests inférentiels (Tableau 4) réalisés au niveau de chacune d'elles ont permis de comparer les valeurs des variables (âge, circonférence et hauteur de coupe) des souches vivantes à celles des souches mortes.

En effet, les valeurs de l'âge au niveau des souches vivantes de Pterocarpus erinaceus ont été significativement inférieures au seuil de $5 \%$ à celles des souches mortes. $\mathrm{Au}$ niveau de Vitellaria paradoxa, les hauteurs de coupe et les circonférences des souches vivantes ont été certes inférieures à celles des souches mortes mais la différence n'est pas significative. Au niveau des souches de Anogeissus leiocarpa en revanche, aucun des facteurs étudiés n'a confirmé l'hypothèse émise sur la base des tendances observées.

Somme toute, ces résultats montrent que l'âge des souches est un facteur hautement déterminant dans la survie des souches de Pterocarpus erinaceus. La hauteur de coupe a aussi un effet à la limite significatif $(6 \%)$. Ainsi, il est possible de prédire à court terme le taux de survie des souches de Pterocarpus erinaceus par un modèle de régression (Tableau 5). 
Le modèle n'est globalement significatif que dans le cas de l'espèce Pterocarpus erinaceus et explique $43 \%$ des variations du taux de survie. Cette analyse ne fait que confirmer les résultats du test inférentiel cidessus réalisé. Mais en plus de l'âge qui était le seul facteur déterminant, la circonférence des souches et la hauteur de coupe ont tous eu de lien significatif avec le taux de survie des souches $(\mathrm{p}<0,05)$. Le modèle ainsi établi a révélé que la survie des souches de Pterocarpus erinaceus est hautement influencée par l'âge et les dimensions des souches. Le signe négatif des coefficients de ces variables confirme que ces dernières influent négativement sur la survie des souches. Dans les modèles obtenus pour Anogeissus leiocarpa et Vitellaria paradoxa, seule la constante est significative. La valeur de cette constante représente le taux fixe de survie qu'il est possible d'obtenir quels que soient l'âge et les dimensions des souches. Ce taux fixe est respectivement égal à 55,01\% chez Anogeissus leiocarpa et 77,26\% chez Vitellaria paradoxa.

Tableau 1 : Inertie des données.

\begin{tabular}{llllll}
\hline Axes & F1 & F2 & F3 & F4 & Inertie totale \\
\hline Inertie & 0,716 & 0,704 & 0,612 & 0,567 & 4,046 \\
Variance (\%) & 17,69 & 17,41 & 15,11 & 14,02 & \\
Variance cumulée (\%) & 17,69 & 35,10 & 50,21 & 64,22 & \\
\hline
\end{tabular}

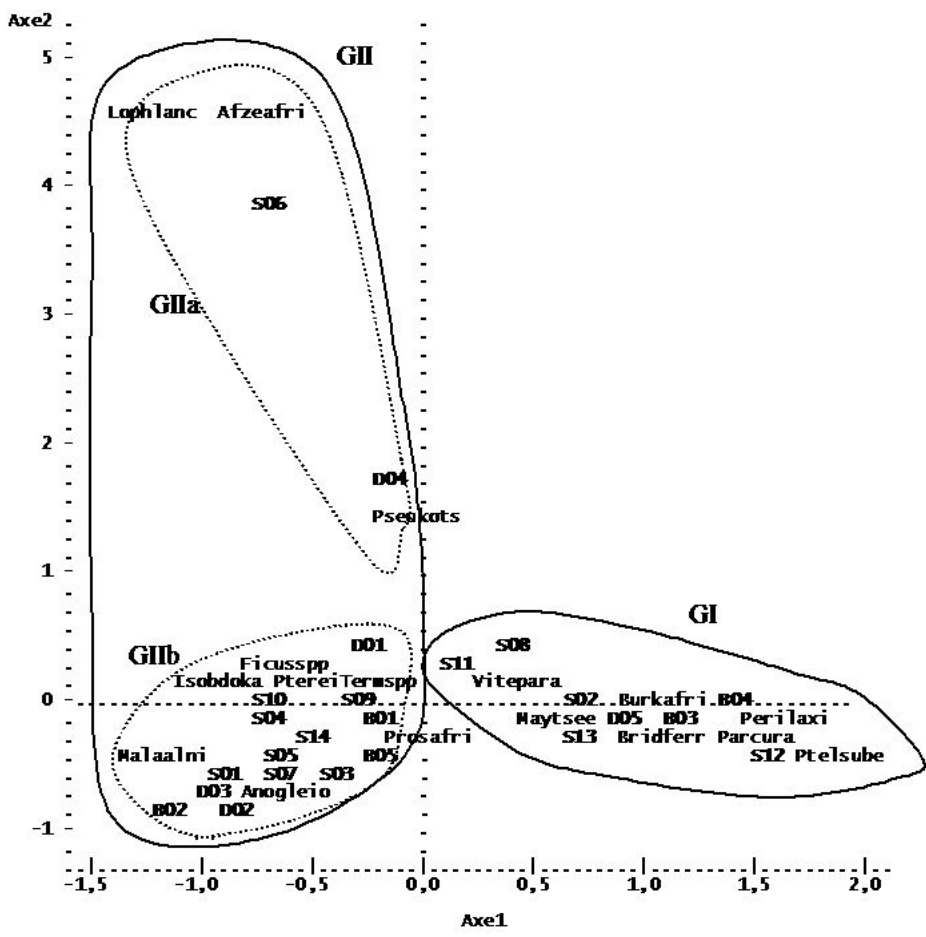

Figure 2: Diagramme de la matrice 24 chantiers x 17 espèces ligneuses exploitées pour la production de charbon de bois. 
A. S. YAOITCHA et al. / Int. J. Biol. Chem. Sci. 10(4): 1702-1716, 2016

Tableau 2 : Analyse des caractéristiques des groupes de chantiers.

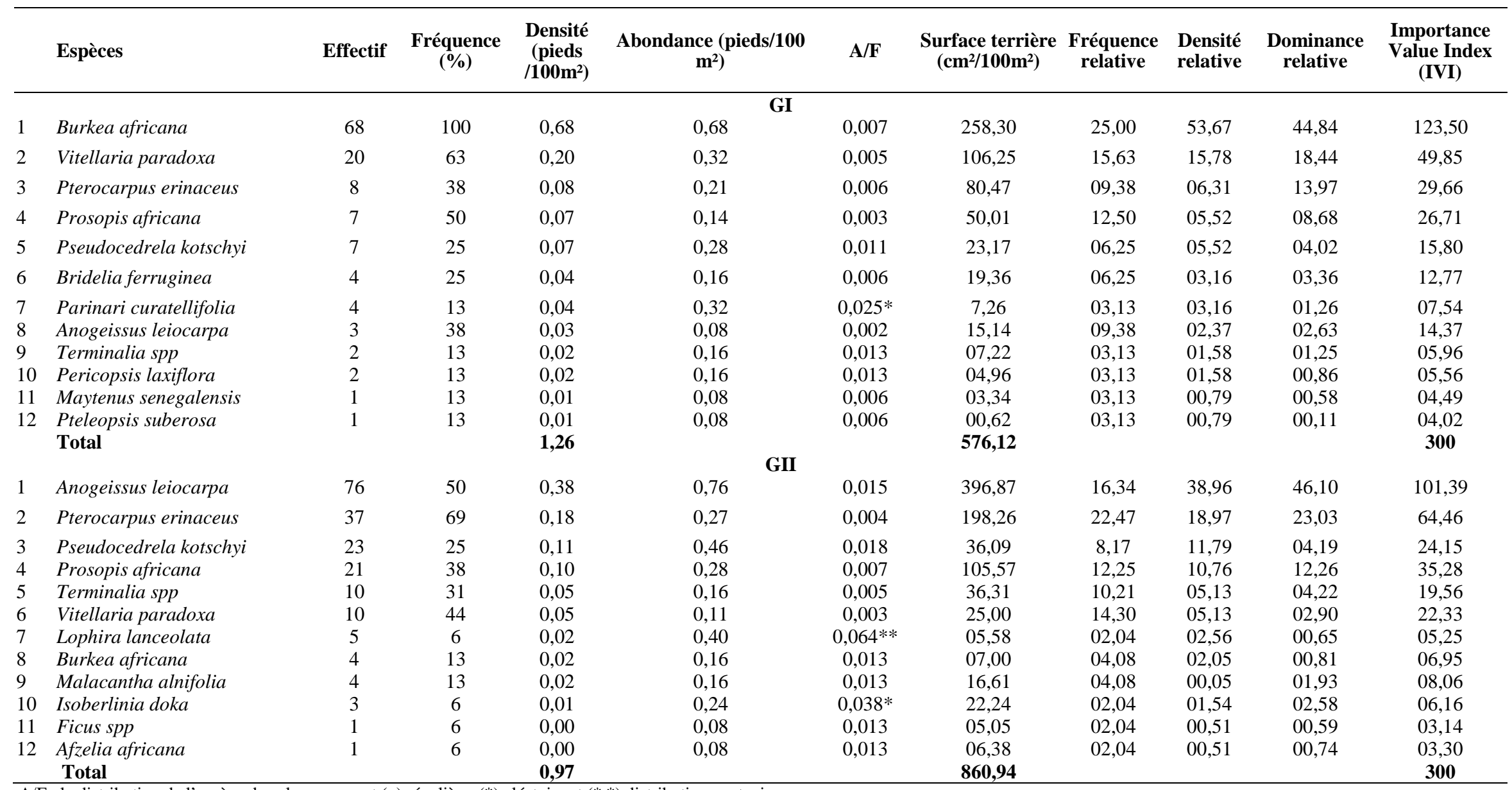

$\mathrm{A} / \mathrm{F}$ : la distribution de l'espèce dans le groupe est $($ ) régulière, $(*)$ aléatoire et $(* *)$ distribution contagieuse 
Tableau 3 : Taux de survie (\%) des souches des espèces.

\begin{tabular}{lccc}
\hline \multirow{2}{*}{ Espèces } & \multicolumn{2}{c}{ Nombre de souches } & Taux (\%) \\
\cline { 2 - 4 } & Totales & Vivantes & Survie \\
\hline Prosopis africana & 28 & 28 & 100,00 \\
Burkea africana & 72 & 71 & 98,61 \\
Pseudocedrela kotschyi & 30 & 29 & 96,67 \\
Pterocarpus erinaceus & 45 & 30 & 66,67 \\
Vitellaria paradoxa & 30 & 20 & 66,67 \\
Anogeissus leiocarpa & 79 & 47 & 59,49 \\
Terminalia spp & 12 & 8 & 66,67 \\
Bridelia ferruginea & 4 & 2 & 50,00 \\
Malacantha alnifolia & 4 & 1 & 25,00 \\
Parinari curatellifolia & 4 & 0 & 0,00 \\
Isoberlinia doka & 3 & 0 & 0,00 \\
Lophira lanceolata & 5 & 5 & 100,00 \\
Pericopsis laxiflora & 2 & 2 & 100,00 \\
Afzelia africana & 1 & 1 & 100,00 \\
Ficus spp & 1 & 1 & 100,00 \\
Maytenus senegalensis & 1 & 1 & 100,00 \\
Pteleopsis suberosa & 1 & 1 & 100,00 \\
Total & $\mathbf{3 2 2}$ & $\mathbf{2 4 7}$ & $\mathbf{7 6 , 7 1}$ \\
\hline
\end{tabular}


Tableau 4 : Tests univariés sur les facteurs de variabilité de la survie des souches des espèces.

\begin{tabular}{llccc}
\hline \multicolumn{1}{c}{ Espèces } & \multicolumn{1}{c}{ Facteur } & \multicolumn{2}{c}{ Moyenne $\pm \boldsymbol{\sigma}$} & \multirow{P}{*}{$>|\mathbf{t}|$} \\
\cline { 3 - 4 } & & Souches vivantes & Souches mortes & \\
\hline Pterocarpus & Age (ans) & $1,8 \pm 0,8$ & $2,60 \pm 0,74$ & $<0,01$ \\
erinaceus & Circonférence $(\mathrm{cm})$ & $106,2 \pm 32,39$ & $120,5 \pm 34,35$ & 0,18 \\
& Hauteur de coupe $(\mathrm{cm})$ & $45,76 \pm 12,30$ & $53,80 \pm 15,06$ & 0,06 \\
Vitellaria & Age (ans) & $2,10 \pm 0,72$ & $2,00 \pm 0,47$ & 0,69 \\
paradoxa & Circonférence $(\mathrm{cm})$ & $70,20 \pm 23,76$ & $88,10 \pm 34,24$ & 0,10 \\
& Hauteur de coupe $(\mathrm{cm})$ & $31,70 \pm 14,43$ & $38,30 \pm 9,20$ & 0,20 \\
Anogeissus & Age (ans) & $2,38 \pm 0,77$ & $2,44 \pm 0,62$ & 0,74 \\
leiocarpa & Circonférence $(\mathrm{cm})$ & $99,66 \pm 58,82$ & $97,28 \pm 54,88$ & 0,86 \\
& Hauteur de coupe $(\mathrm{cm})$ & $57,74 \pm 26,69$ & $57,47 \pm 19,22$ & 0,96 \\
\hline
\end{tabular}

Tableau 5 : Modèle de régression linéaire du taux de survie des souches.

\begin{tabular}{llcccc}
\hline Espèces & Facteur & $\mathbf{R}^{\mathbf{2}}$ & $\begin{array}{c}\text { Paramètres } \\
\text { estimés }\end{array}$ & $\mathbf{F}$ & $\mathbf{P}>\mathbf{F}$ \\
\hline \multirow{3}{*}{$\begin{array}{l}\text { Pterocarpus } \\
\text { erinaceus }\end{array}$} & Modèle & $\mathbf{0 , 4 3 1 0}$ & & $\mathbf{1 0 , 1 0}$ & $<\mathbf{0 , 0 0 0 1}$ \\
& Constante & & 2,07794 & 53,73 & $<0,0001$ \\
& Age (ans) & & $-0,24625$ & 17,37 & 0,0002 \\
& Circonférence $(\mathrm{cm})$ & & $-0,00411$ & 7,35 & 0,0098 \\
& Hauteur de coupe $(\mathrm{cm})$ & & $-0,00881$ & 5,27 & 0,0270 \\
& Modèle & $\mathbf{0 , 0 4 3 5}$ & & $\mathbf{1 , 1 4}$ & $\mathbf{0 , 3 3 9 5}$ \\
Anogeissus & Constante & & 0,55013 & 10,13 & 0,0021 \\
Leiocarpa & Age (ans) & & $-0,03622$ & 0,37 & 0,5459 \\
& Circonférence $(\mathrm{cm})$ & & 0,00129 & 3,17 & 0,0789 \\
& Hauteur de coupe $(\mathrm{cm})$ & & 0,00008 & 0,00 & 0,9634 \\
& Modèle & $\mathbf{0 , 0 5 1 3}$ & & $\mathbf{0 , 4 5}$ & $\mathbf{0 , 7 1 8 8}$ \\
Vitellaria & Constante & & 0,77257 & 4,43 & 0,0456 \\
Paradoxa & Age (ans) & & 0,03406 & 0,09 & 0,7681 \\
& Circonférence $(\mathrm{cm})$ & & $-0,00279$ & 1,08 & 0,3088 \\
& Hauteur de coupe $(\mathrm{cm})$ & & 0,00060 & 0,01 & 0,9135 \\
\hline
\end{tabular}




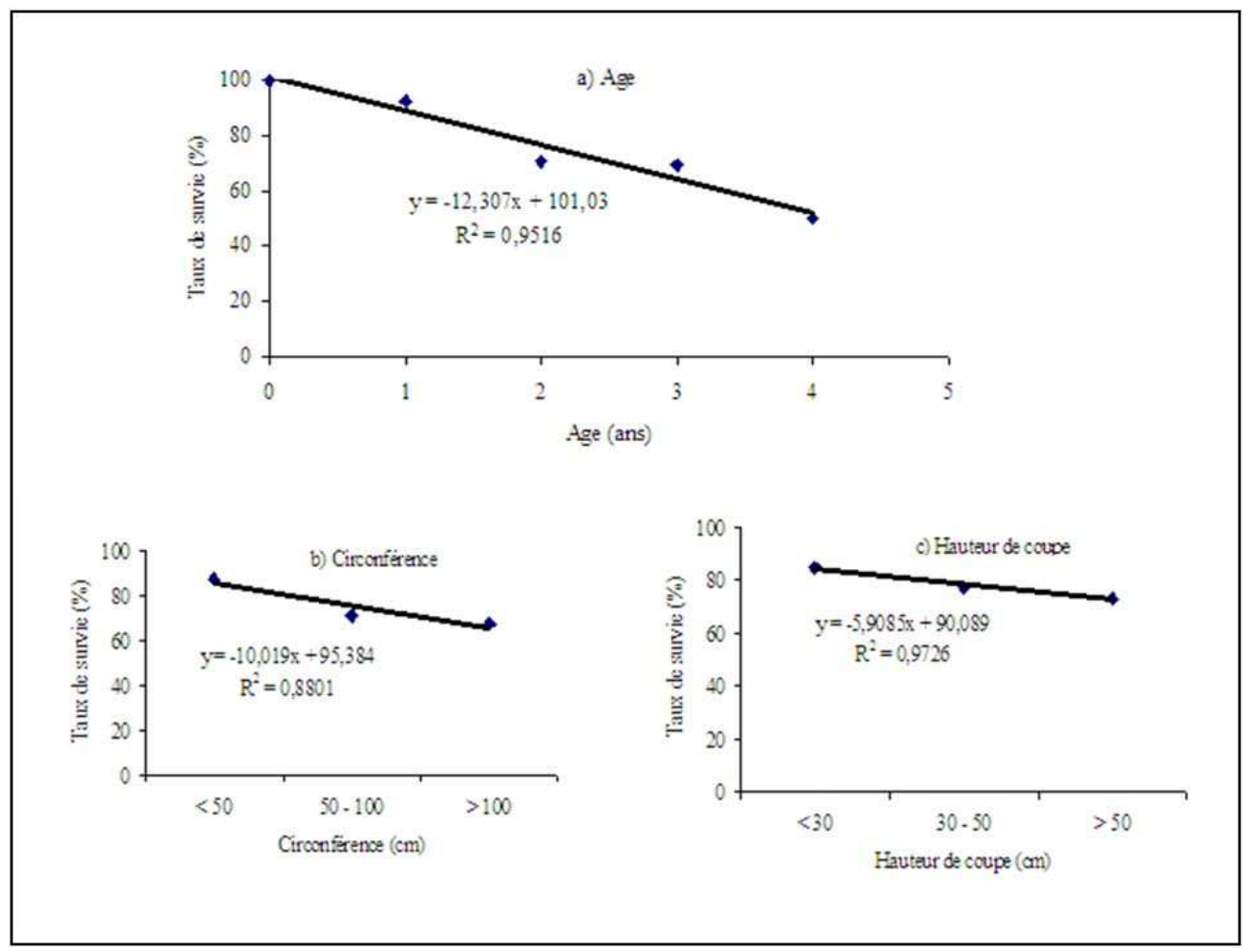

Figure 3 : Evolution du taux de survie des souches de l'ensemble des espèces en fonction de l'âge, de la circonférence et de la hauteur de coupe.

\section{DISCUSSION}

\section{Cortège des espèces exploitées pour la} production du charbon

Les principales souches d'espèces rencontrées ont été Burkea africana, Prosopis africana, Anogeissus leiocarpa, Pterocarpus erinaceus, Vitellaria paradoxa et Pseudocedrela kotschyi. Par ordre d'importance, Anogeissus leiocarpa, Prosopis africana, Burkea africana et Pterocarpus erinaceus; ont été les plus exploitées et plus recherchées pour leur qualité et leur rendement dans la production de charbon. Ainsi, l'installation d'un chantier de production dans une formation va dépendre de sa richesse en ces quatre espèces. Les premiers chantiers de charbon ont été donc installés dans les peuplements dominés par l'une de ces espèces. Toutefois, Anogeissus leiocarpa et Burkea africana ont été les seules parmi ces espèces qui s'établissent en population. Quoique, les peuplements à Burkea africana se retrouvent plus rarement ou sur des étendues plus restreintes, en raison de la rareté des sites correspondants à son exigence (Arbonnier, 2000; Witting et al., 2002). Pterocarpus erinaceus et Anogeissus leiocarpa ont été retrouvées le plus souvent à l'état isolé ou en nombre relativement faible dans les peuplements. Ainsi, les arbres de Burkea africana ont été donc exploités sur les mêmes stations avec beaucoup d'autres espèces, tandis que Anogeissus leiocarpa reste pratiquement la seule espèce dont l'exploitation se fait dans sa station en raison de sa grande étendue. C'est ce qui explique la formation des deux groupes: groupe de chantiers à Burkea africana et Parinari curatellifolia (GI), constitué des chantiers sur lesquels une large gamme d'espèces ont été exploitées et le groupe GII constitué des sousgroupes de chantiers à Anogeissus leiocarpa et Isoberlinia doka (GIIb) et à Pseudocedrela kotschyi et Lophira lanceolata (GIIa) constitué de chantiers quasi-monospécifiques.

Ces espèces autrefois très communes, ont été de nos jours décimées et sont devenues très rares dans les formations naturelles. En 
conséquence, plusieurs autres espèces de faible ou moyen potentiel de carbonisation ont été identifiées par les exploitants pour les remplacer. Il s'agit de : Vitellaria paradoxa et Pseudocedrela kotschyi, toutes deux fréquentes dans la zone soudanienne. Elles sont surtout exploitées pour leur rendement élevé en charbon. Aussi, tous les arbres du genre Terminalia sont-ils abattus non pas pour la qualité du charbon extrait mais pour leur aptitude à donner un bon rendement. Cela confirme l'idée émise par Schneider (1996) et Sieglstetter et Witting (2002) selon laquelle la disponibilité est un facteur déterminant dans le choix des espèces utilisées comme bois de chauffe. Les arbres de plusieurs autres espèces peu fréquentes dans les formations sont également abattus pour la même cause (Bridelia ferruginea, Parinari curatellifolia, Pericopsis laxiflora, Pteleopsis suberosa, Ficus sp, Isoberlinia doka, Malacantha alnifolia, Maytenus senegalensis, Lophira lanceolata). Cela indique que l'abattage des espèces utilisées dans la production du charbon n'est point sélectif et que toutes les espèces sont bonnes à être exploitées pourvu qu'elles donnent du charbon. Par ailleurs, la liste des espèces utilisées pour la production du charbon au Bénin pourrait être améliorée par d'autres études plus approfondies étendues sur tout le territoire national. Des études pouvant fournir des informations sur la nature et la qualité des sujets épargnés au cours des abattages à travers des relevés globaux sur les chantiers sont vivement recommandées.

\section{Facteurs de variabilité de la survie des souches}

Parmi les principales espèces exploitées, deux catégories d'espèces ont été identifiées en matière de régénération des souches : celle des espèces dont la survie des souches est assurée à environ 100\% quels que soient l'âge et les dimensions des souches et celle des espèces dont la survie est variable. On compte dans la première catégorie deux espèces de la famille des Leguminosae: Prosopis africana et Burkea africana. Nous excluons de cette dernière, Pseudocedrela kotschyi à cause de l'âge des souches qui est assez récent ( $0-1$ an). Ainsi, l'on peut croire que les légumineuses sont qualifiées d'espèces dont toutes souches donnent de rejets à $100 \%$. Des travaux de Sawadogo et al. (2002) ont rapporté que dans la zone soudanaise au Burkina Faso, la survie des souches des espèces de la famille des Leguminosae pouvait aussi diminuer jusqu'au 50\% Piliostigma thonningii voire moins de $10 \%$ Entada africana et Detarium microcarpum. Aussi, avons-nous eu une légumineuse, Pterocarpus erinaceus qui fait partie de la seconde catégorie des espèces dont les souches ne portent pas toutes de rejets ou sont mortes. Cette seconde catégorie d'espèces est constituée en plus de Pterocarpus erinaceus, des espèces telles que Anogeissus leiocarpa et Vitellaria paradoxa. Leur survie est variable en fonction de l'âge et des dimensions des souches (grosseur et hauteur de coupe).

De façon empirique, il est connu que toutes les souches d'arbre sont vivantes après la coupe et sont toutes susceptibles de régénérer. Mais au fil des ans, certaines d'entre elles peuvent mourir sous l'effet de défrichement dans les champs (Faye, 2000), des feux (Yaoitcha, 2004) et du broutage (Sawadogo et al., 2002). Par ailleurs, le modèle prédictif de la survie des souches des espèces a révélé des relations négatives entre les taux de survie des souches et leurs dimensions notamment chez Pterocarpus erinaceus. Cependant, les résultats tirés des travaux de Luoga et al. (2004), Shackleton, (2001), Kaschula et al. (2005), ont montré que les grosses et/ou les hautes souches sont plus productives en rejets (en nombre et en taille) que les petites et/ou basses souches. Le cas de Pterocarpus erinaceus peut s'expliquer par le fait qu'elle soit un ligneux fourrager et ses repousses sont très appétées par les bovins. En effet, les rejets produits par les grosses souches de Pterocarpus erinaceus attireraient les bovins qui y exerceraient une forte pression. De façon récidive, il en résultera une baisse drastique de production de rejets. A long ou à moyen terme, les souches s'épuiseront totalement de leurs réserves. Elles perdront l'expression de leur potentiel d'émission de rejets et finiront par en mourir. La régression ainsi établie met en évidence la menace qui pèse sur Pterocarpus erinaceus dans ce contexte d'exploitation massive pour la production $\mathrm{du}$ charbon. Le potentiel 
d'émission des rejets des souches s'amenuise au fil des ans et si les coupes des arbres de cette espèce ne cessent, on assistera à la disparition de cette dernière à long terme. Par ailleurs, cette menace est beaucoup plus grande pour peu qu'elle fasse l'objet d'émondage par les peulh pasteurs fougueux à nourrir leurs troupeaux en saison sèche (Houinato et Sinsin, 2000). Quant aux autres espèces que sont Anogeissus leiocarpa et Vitellaria paradoxa, les mortalités enregistrées n'ont été significativement liées à aucun des facteurs étudiés et peuvent être soit liées à d'autres facteurs que des études plus approfondies nous permettront d'identifier. Néanmoins les taux de mortalité enregistrés $(11,11 \%$ - 45\%) sont dans les mêmes ordres de grandeur que ceux observés par certains auteurs (Fall et al., 2000 ; Sawadogo et al., 2002) pour les espèces telles que Acacia seyal, A. mellifera, Bauhinia rufescens, Ziziphus mauritiana, Combretum glutinosum, Anogeissus leiocarpa et Piliostigma thonningii. Il n'y a pas encore péril en la demeure quant à la gestion de leur régénération. Toutefois, des études axées sur les facteurs déterminants de la régénération et de la survie des souches des essences d'une part et la contribution du semis naturel à la régénération des espèces les plus exploitées pour la production du charbon de bois d'autre part restent à réaliser pour la mise au point efficace des outils de conservation de ces espèces menacées.

\section{Conclusion}

Le présent travail a permis de déterminer les principales espèces exploitées pour la production du charbon et des facteurs influençant la survie des souches. Par ordre d'importance, les espèces exploitées sont Anogeissus leiocarpa, Prosopis africana, Burkea africana et Pterocarpus erinaceus. En matière de la régénération, la survie des souches de certaines espèces fortement recherchées tels que Pterocarpus erinaceus, Anogeissus leiocarpa et Vitellaria paradoxa est assujettie aux variations de l'âge et les dimensions des souches. Des stratégies de conservation relatives à la promotion de l'agroforesterie, aux plantations, au renforcement des capacités des producteurs sur les techniques alternatives de production du charbon et à la politique de subvention du gaz domestique peuvent être déjà développées pour mieux assurer l'utilisation durable de toutes les espèces qui sont devenues la proie de la production du charbon au Bénin.

\section{REMERCIEMENTS}

Nous remercions l'Institut National des Recherches Agricoles du Bénin (INRAB) et le projet AGRAN pour nous avoir accordé le financement pour l'exécution des travaux de recherche et l'Agence Universitaire Francophone (AUF) pour avoir accordé à YAOITCHA Alain Sèakpo la bourse de mobilité pour son séjour au Laboratoire d'Ecologie à la Faculté des Sciences et Techniques de l'Université Cheikh Anta Diop de Dakar.

\section{CONFLIT D'INTERETS}

Les auteurs déclarent n'avoir aucun conflit d'intérêts.

\section{CONTRIBUTIONS DES AUTEURS}

ASY a rédigé le protocole de recherche, a collecté les données et a rédigé le manuscrit ; ABA a contribué à l'amélioration du protocole de recherche et a collecté les données ; AGZ a contribué à l'amélioration du manuscrit ; $\mathrm{MH}$ a contribué à l'amélioration du manuscrit; GAM a contribué à l'amélioration du protocole de recherche ; BS a supervisé les travaux de recherche ; ELA a offert le cadre pour la rédaction du manuscrit et a contribué à l'analyse des données.

\section{REFERENCES}

Agbani PO, Sinsin B, Hahn-Hadjal K. 2004. Phytodiversity Decline: The local perception in the soudan zone of norh West Bénin. 5th inter Symposium of Tropical African Biodiversity-Molecule, Ortganisme-ecosystem, Bon 2 - 6 may 2004.

Arbonnier M. 2000. Arbres, Arbustes et Lianes des Zones Sèches d'Afrique de l'Ouest. CIRAD - MNHN - UICN.

Bellefontaine R, Edelin C, Ichaou A, du Laurens D, Monsarrat A, Loquai C. 2000. Le drageonnage, alternative aux semis et aux plantations de ligneux dans 
les zones semi-arides : protocole de recherches. Secheresse, 4(11): 221-226.

Bellefontaine R. 2005. Stratégies de régénération, de survie, d'occupation de l'espace de 990 espèces ligneuses. Sécheresse en ligne vol. $1 \mathrm{E}, \mathrm{n}^{\circ} 3$, décembre 2005. http://www.secheresse.info/article.php3 ?id_article=2344.

Chabot-Boivin S, Margolis AH, Weber CJ. 2004. Variation in coppice-shoot growth among provenances of Calycophyllum spruceanum Benth. in the Peruvian Amazon Basin. For. Ecol. Man., 198: 249-260.

Curtis JT, Cottam G. 1956. Plant Ecology Work Book. Laboratory Fiel Reference Manuel. Burgess Publishing Co. Minnisota.

Djohossou P. 2000. Rapport national sur le secteur forestier au Bénin, Annexe VIII. In: Actes De L'atelier Sous-Regional pour Sous-Région Ecowas sur la collecte et analyse des données forestières / proceedings of sub regional workshop on forestry statistics and outlook study for Africa/Fosa, Yamoussoukro, Côte d'Ivoire, 13-18 Décembre 1999, Programme de partenariat CE-FAO/EC-FAO partnership programme.

Dourma M, Guelly KA, Kokou K, Batawila K, Wala K. Bellefontaine R, Akpagana K. 2006. Importance de la multiplication végétative par drageonnage de deux espèces d'Isoberlinia dans les formations soudaniennes du Nord-Togo. Bois et Forêts des Tropiques, $\mathrm{n}^{\circ} \mathbf{2 8 9}$, $3^{\text {ème }}$ trimestre 2006.

Dubroeucq D. 1997. Note Explicative $N^{\circ} 66$ (3). Carte Pédologique de Reconnaissance de la République Populaire du Bénin à 1/200 000. Feuille de Savè. ORSTOM, Paris, 45 p.

Fall ST, N'diaye SA, Traoré E. 2000. Exploitation des arbres à usages multiples dans les systèmes d'élevage des zones soudanienne et sahélienne (Sénégal) in G. Gintzburger, M. Bounejmate, C. Agola and K. Mossi (eds.). 2000. Production and Utilization of Multi-purpose Fodder Shrubs and
Trees in West Asia, North Africa and the Sahel. ICARDA, Aleppo, Syria; ILRI, Narobi, Kenya. viii+ 60 pp. http://www.ilri.cgiar.org/InfoServ/Webp ub/Fulldocs/FodShrub/SENEGAL.htm

FAO. 2000. Situation des forêts et de la faune sauvage en Afrique. $12^{\text {ème }}$ session de la commission des forêts et de la faune sauvage pour l'Afrique. Lusaka (Zambie), $27 \quad$ - 30 mars 2000. http://www.fao.org/docrep/meeting/x42 01f.htm

Faye EH. 2000. Etude de la dynamique des souches ligneuses dans le cycle culturejachère en zone soudanienne. Mém. Ing. Dév. Rur. IDR/UPBD/Burkina Faso, p 103.

Forrester D, Bauhus J, Connell M. 2003. Competition in thinned Silvertop Ash (Eucalyptus sieberi L. Johnson) stands from early coppice growth). For. Ecol. Man., 174: 459-475.

Harrington, RA, Fownes, J.H, 1995. Radiation interception and growth of planted and coppice stands of four fast-growing tropical trees. Journal of Applied Ecology, 32: 1-8.

Houinato M, Sinsin B. 2000. La pression agro-pastorale sur la Zone Riveraine de la Réserve de la Biosphère de la Pendjari. Tropicultura, 18(3): 112-117.

Kabore C. 2002. Aménagement des Forêts du Sahel. Point sur les Vingt Années de Pratiques au Burkina Faso. Direction Générale des Eaux et Forêts, Cellule Stratégie et Méthode, CSM/DGEF : Ouagadougou ; p 139.

Kaschula SA, Twine WC, Scholes MC. 2005. Foundation for environmental conservation the effect of catena position and stump characteristics on the coppice response of three savannah fuelwood species. Environmental Conservation, 32 (1): 76-84.

Luoga, EJ, Witkowski, ETF, K. Balkwill. 2004. Regeneration by coppicing (resprouting) of miombo (African savanna) trees in relation to land use. Forest Ecology and Management, 189: 23-35.

MEPN (Ministère de l'Environnement et de la Protection de la Nature) 2009. $4^{\text {ème }}$ 
Rapport National du Bénin sur la Convention des Nations Unies sur la Diversité Biologique, PNUD/MEPN, Cotonou, Bénin, p 151.

PBF II/ IFN (Projet Bois de Feu phase II/ Inventaire forestier National) 2007. Bassins d'approvisionnement en boisénergie de Cotonou, Porto Novo, Lokossa, Abomey, Bohicon, Djougou, Natitingou et Parakou, Rapport de mission de Claudine SERRE DUHEM Mission au Bénin du 6 au 25 juin 2007, Ministère de l'Environnement et de la Protection de la Nature, Cotonou, Bénin p 54.

Sawadogo L, Nygard R, Pallo F. 2002. Effects of livestock and prescribed fire on coppice growth after selective cutting of Sudanian savannah in Burkina Faso. Ann. For. Sci., 59: 185-195.

Schneider, PD. 1996. Sauvegarde et aménagement de la forêt de Farako (Région de Sikasso, Mali-Sud) avec la participation et au profit des populations riveraines. - Thèse EPFZ $\mathrm{n}^{\circ} 11867$ Zürich, $322 \mathrm{p}$.

Shackleton CM. 2000. Stump size and the number of coppice shoots for selected savanna tree species. South African Journal of Botany, 66: 124-127.

Shackleton CM. 2001. Managing regrowth of an indigenous savanna tree species (Terminalia sericea) for fuelwood: the influence of stump dimensions and posthavest coppice pruning. Biomass and Bioenergy, 20: 261-270.

Shannon CE, Weaner W. 1963. The Mathmatical Theory of Comminication. University of Illinois Press: Usbana.

Sieglstetter R, Witting R. 2002. L'utilisation des ligneux sauvages et son effet sur la végétation dans la région d'Atakora (Bénin nord-occidental). Etudes flor. Vég. Burkina Faso, 7: 23-30.

Sims EHR, Maiava GT, Bullock TB. 2001. Shoort rotation coppice tree species selection for woody biomass production in New Zealand. Biomass and Bioenergy, 20: 329-335.

Tchiwanou M. 2003. Impact de l'exploitation illégale $\mathrm{du}$ bois énergie sur la dégradation des ressources naturelles et l'aggravation de la pauvreté rurale au Bénin. In : Conférence ministérielles sur l'application des lois forestières et l'amélioration de la gouvernance, dans le secteur forestier en Afrique, Yaoundé du 13 - 16 octobre 2003 ;

White F. 1983. The vegetation of Africa. UNESCO, Paris, p 35.

Witting R, Hann-Hadjali K, Krormer J, Müller J, Sieglstetter R. 2002. La végétation actuelle des savanes du Burkina Faso et du Bénin - sa signification pour l'homme et la modification pour l'homme (aperçu des résultats d'un projet de recherche duré des années). Etudes flor. Vég. Burkina Faso, 7: 0316.

Yaoitcha AS. 2004. Dynamique des pâturages naturels suivis de 2000 à 2004 sur les fermes d'élevage de Bétécoucou et de Samiondji et l'exploitation pastorale des alentours par les éleveurs bovins riverains. Mém. Ing. Agro. FSA/UAC/Bénin. p 111.

Yaoitcha AS. 2007. Potentiel de régénération des chantiers de production du charbon de bois au Centre-Bénin. Mém. DEA. FSA/UAC/Bénin. p 33. 